\title{
Planning Oral Health and Clinical Discharge in Primary Care: The Comprehensive Dental Care Protocol Outcome
}

\author{
${ }^{1}$ Yuri Wanderley Cavalcanti, ${ }^{2}$ Leopoldina de Fátima Dantas de Almeida
}

${ }^{3}$ Ailma de Souza Barbosa, ${ }^{4}$ Wilton Wilney Nascimento Padilha

\begin{abstract}
Introduction: The dental care must be driven by preventive and curative measures that can contribute to the population's oral health promotion.
\end{abstract}

Objective: To evaluate the impact of the actions proposed by a comprehensive dental care protocol (CDCP) on the oral health condition of primary care users.

Materials and methods: The sample consisted of 32 volunteers, assisted throughout the six phases proposed by the CDCP: diagnosis of dental needs; resolution of urgencies; restorative interventions; application of promotional measures; evaluation of the achieved health level; and periodic controls. Data were collected through clinical exams, which measured the simplified oral hygiene index (OHI-S), gingival bleeding index (GBI) and the decayed, missing and filled teeth (DMFT) Index, before and after the CDCP was implemented. Statistical analysis consisted of the Wilcoxon test, at $5 \%$ significance level $(\alpha=0.05)$

Results: The $\mathrm{OHI}-\mathrm{S}$ and $\mathrm{GBI}$ indices showed a significant reduction $(p<0.05)$ from the initial $(1.4 \pm 0.6$ and $46.3 \pm 19.9)$ to final condition $(0.9 \pm 0.3$ and $21.5 \pm 7.5)$. The decayed, missing and filled teeth and the missing teeth component were not significantly altered $(p>0.05)$, showing final values equal to $12.7 \pm 9.6$ and $5.6 \pm 7.8$, respectively. Decayed elements were fully converted into filled elements, and the final values of the decayed and filled elements were, respectively, $0.0 \pm 0.0$ and $7.3 \pm 5.7(p<0.05)$.

Conclusions: The enactment of the CDCP had a beneficial effect on the oral health of the population assisted by the dental services offered in primary care and this protocol seems to fit the public dental service demands.

Clinical significance: The CDCP can be useful to public dental service planning since it showed an efficient clinical outcome to

${ }^{1}$ Department of Prosthodontics and Periodontology, Piracicaba Dental School, University of Campinas, Piracicaba, Brazil

${ }^{2}$ Department of Restorative Dentistry, Araraquara Dental School, University of São Paulo, Araraquara, Brazil

${ }^{3}$ Department of Dentistry, Public Dental Service in Primary Care, Unified Health System, João Pessoa, Brazil

${ }^{4}$ Department of Dental Clinics and Community Dentistry Federal University of Paraíba, João Pessoa, Brazil

Corresponding Author: Yuri Wanderley Cavalcanti, PhD Student, Department of Prosthodontics and Periodontology Piracicaba, Dental School, University of Campinas, PO Box 52, ZIP-Code: 13414-903, Piracicaba, São Paulo, Brazil, Fax: +55 192106 5295, e-mail: yuri.wanderley@yahoo.com.br the patients. We consider that this protocol should be employed in primary care oral health services in order to achieve overall upgrade, access enlargement and public oral health promotion.

Keywords: Comprehensive dental care, Oral health, Primary healthcare.

How to cite this article: Cavalcanti YW, de Fátima Dantas de Almeida L, de Souza Barbosa A, Padilha WWN. Planning Oral Health and Clinical Discharge in Primary Care: The Comprehensive Dental Care Protocol Outcome. J Contemp Dent Pract 2015;16(3):172-177.

Source of support: Nil

Conflict of interest: None

\section{INTRODUCTION}

Primary healthcare in Brazil's public sector, represented by the country's unified health system (SUS), is responsible for offering actions and services to valorize prevention, promotion, treatment and recovery of the population health. At this level of care, the family is seen as the primary social nucleus, with value given to universal access, humanized assistance, teamwork, links between healthcare professionals and the community, integrality and enhanced case resolution. ${ }^{1}$ With that, SUS aims to develop integral, resolutive and participative care, capable of having an impact and solving the main (and most urgent) health problems among the population.

In the scope of oral health, the Smiling Brazil Program, which began in 2004, has favored improvements in Dentistry at the basic care level through financing, expansion of services and incorporation of the principles and guidelines that drives SUS. Nevertheless, the proposal of adequate intervention programs for the context of the communities at which they are directed represents an obstacle to be overcome by the public health system. ${ }^{2}$

The epidemiological profile of oral disease in Brazil shows a reduction in the rates of cavities and tooth extractions with a concomitant increase in restorative and rehabilitative treatments offered to the population. However, access to health services still shows limitations, characterized by underuse of preventive actions. ${ }^{3}$

Historically, the prevailing dental care model in Brazil has been characterized by a lack of action planning and segmentation among the different specialties. The 
existing individualism of dental practice is evidenced by the curative-restorative assistance model, offered by a single professional under a specialty perspective, directed at ailing individuals. This traditional model features other characteristics, such as reduced access, lack of humanization, absence of integrality and a technical character for all actions. Therefore, service is not organized based on the standpoint of the patient/ user, but rather under the perspective of profit and the market, influencing the public service network as well. ${ }^{4}$

While considering the diagnosis and costs of treatment plans established by dental surgeons of the SUS, a trend was observed towards curative-restorative interventions, even when unnecessary. ${ }^{5}$ This aspect reveals the negative impact of that professional practice on the oral healthcare of the population and on the cost of public health services. $^{5}$

Thus, the formulation of a care proposal that meets the demands of the public dental service must consider the identification and resolution of basic oral health needs, humanized assistance, and proper referral to the adequate specialty service, incorporation of self-care practices by patients, regularly scheduled controls, health promotion and high clinical discharge rates. Moreover, the development of a proposal for qualification of this sector must be accompanied by an adequate evaluation process, justifying this study.

The objective of this work was to evaluate the impact of actions proposed in a comprehensive dental care protocol $(\mathrm{CDCP})$ on the oral health of primary care users in the city of Joao Pessoa, Paraiba, Brazil.

\section{MATERIALS AND METHODS}

An intervention study was performed using the action research method, at a primary healthcare unit in the city of Joao Pessoa, Paraiba, Brazil. Care was given weekly to users of the health service, for a 6 months period, by a single trained researcher, supported by the research team.

The present study was approved by the Research Ethics Committee of the Paraiba State Health Secretariat, under protocol CAAE 1203.349-2010. Participants in the study were informed with regard to the ethical aspects and voluntarily signed the free and informed consent form (FICF). All international ethics guidelines for biomedical studies involving human beings were respected.

The sample universe consisted of the users registered at the health unit in which the study was developed. As inclusion criteria, participants of the sample had to be in need of dental treatment, be 18 years or older, and accept taking part in the research by signing the FICF. Exclusion criteria consisted of: residing in an area not covered by the health unit, refusing participation in the CDCP or hindering its performance. Out of all individuals enrolled in the dental service $(n=334)$ who agreed to take part in the implementation of the $\mathrm{CDCP}$, a randomized sample was defined after pilot study and sample size calculation from difference between means. Sample size $(n=32)$ yielded an adequate power $(\beta>80 \%)$ to detect statistically relevant significant differences.

The CDCP adopted in this study was based on dental care proposals for basic care clinics. ${ }^{6,7}$ The CDCP established six consecutive phases for each participant, namely: diagnosis of oral health needs; resolution of urgencies; curative-restorative interventions; application of preventive-promotional measures; evaluation of the achieved health level; and periodical controls. To illustrate the implementation phases of the CDCP, a flow chart was devised of the work process developed by the research team (Flow chart 1 ).

The CDCP was developed for a primary care oral health service, with the aim to expand access to the service, solve urgencies, diagnose dental needs, control pain and infection, perform restorative treatments, conduct health education, apply preventive-promotional measures, eliminate health needs, conclude treatments and achieve health promotion. The CDCP was then used as a routine police in the primary care dental service, after pilot studies. The combination of curative-restorative and preventive-promotional measures, as well control and periodic evaluation of the oral environment, represented the means to achieve basic discharge for users at the SUS primary care level. Whenever specialized dental treatment was required, users were referred to SUS reference services.

The concept of basis discharge adopted in this study consists of the absence of need for further preventive or curative dental interventions. ${ }^{6,7}$ Thus, basic discharge was achieved whenever the user's dental treatment needs and oral health measures were efficient. Health education measures and reevaluation of oral levels were performed after Basic Discharge, with monitoring of oral conditions every 6 months and maintaining achieved health levels.

The oral health condition of study participants during the diagnosis and control was evaluated through the simplified oral hygiene index $(\mathrm{OHI}-\mathrm{S}){ }^{8}$, gingival bleeding index (GBI), ${ }^{9}$ and the decayed, missing and filled teeth (DMFT) Index. ${ }^{10}$ Oral indexes were measured by a single trained examiner, resulting in the following Kappa intraexaminer coefficients: 0.73 for IHO-S; 0.70 for GBI; 0.93 for DMFT; and 0.93 for need for treatment.

The data were arranged and analyzed by inferential and descriptive statistics using GraphPad Prism 5.0 soft- 
Flow Chart 1: The developed work process, starting with implementation of the comprehensive dental care protocol Comprehensive Dental Care Protocol*
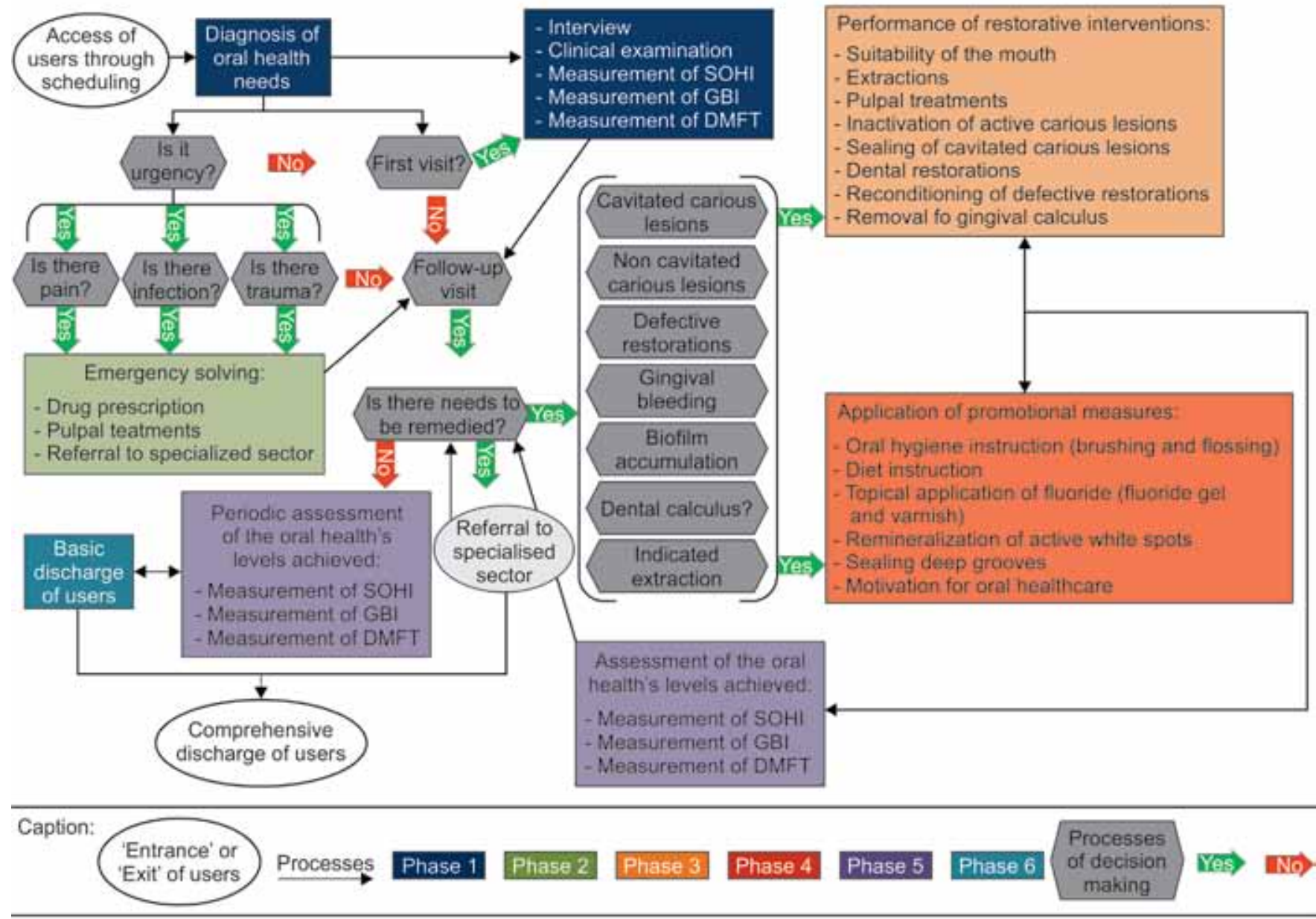

"Proposal developed by the research group on pediatric dentistry and integrated clinic to perform the research project: 'oral health in primary care: comprehensive dental care protocol'

ware (San Diego, CA-USA). Data on the characterization of the sample, total consultations and hours worked, performed procedures, total referrals to specialized sectors, and other data on CDCP productivity were analyzed by descriptive statistics. All data from the evaluation of oral health condition were regarded as nonparametric, and were analyzed using the Wilcoxon test (5\% significance level). The sample size and all the standard deviation yielded an adequate power $(\beta>80 \%)$ to detect statistically relevant significant differences for before and after comparisons.

\section{RESULTS}

Of all study participants, 20 (62.5\%) were female and 12 $(37.5 \%)$ were male. All sampled subjects required dental treatment and oral healthcare, receiving at least three consultations prior to achieving basic discharge.

Of the 32 study participants, 20 received basic discharge, 6 remained under care, and 6 did not complete the established phases of the CDCP. The reason for giving up assistance was changing addresses to areas not covered by the health unit. The individuals who withdrew from the CDCP were referred to the dental service of the appropriate oral health units responsible for dental care coverage. Those who completed the treatment were followed-up by the primary care oral health team, so that basic discharge was expected by continuing with the CDCP at the health service.

During the study period, 120 work hours were spent, over 30, four-hour shifts. During that period, 97 dental appointments were carried out, with 32 initial consultations. A total of 518 basic care oral health procedures were performed, of which 269 (51.93\%) were preventive and promotional, while 249 (48.07\%) were curative and restorative (Table 1). The procedures 'initial dental appointment' and 'referral to the specialized sector' were regarded as promotional because they represent an offer of access to dental service. Referrals to the specialized dental sector of SUS $(n=26)$ consisted of X-ray requests $(n=11)$, endodontic treatments $(n=5)$ and denture fittings $(n=10)$. 
Table 1: Basic curative and restorative dental procedures performed during the first semester of implementation of the comprehensive dental care protocol (Joao Pessoa, 2011)

\begin{tabular}{lll}
\hline Procedure & Number & Percentage (\%) \\
\hline Initial dental appointment & 32 & 6.18 \\
Evaluation of oral indexes & 58 & 11.20 \\
Topical fluoride application (per dental arch) & 40 & 7.72 \\
Action in oral health education & 54 & 10.42 \\
Prescription of topical antimicrobial & 08 & 1.55 \\
Professional prophylaxis & 51 & 9.84 \\
Referral to specialized sector & 26 & 5.02 \\
\hline Total of preventive and promotional procedures & 269 & 51.93 \\
\hline Periodontal scraping (per sextant) & 144 & 27.80 \\
Extraction & 26 & 5.02 \\
Temporary restoration & 11 & 2.12 \\
Dental filling repair & 11 & 2.12 \\
Pulp-dentin protection & 09 & 1.74 \\
Amalgam filling & 20 & 3.87 \\
Composite resin fillings & 28 & 5.40 \\
Curative and restorative procedures & 249 & 48.07 \\
\hline Total performed procedures & 518 & 100.00 \\
\hline
\end{tabular}

Table 2: Means, standard deviations and statistical differences obtained for indexes IHO-S, GBI and DMFT as measured at the initial and final phases of the comprehensive dental care protocol (Joao Pessoa, 2010)

\begin{tabular}{lll}
\hline Indexes & Means and SD & $\begin{array}{l}\text { Statistical differences } \\
\text { between the means }(p)\end{array}$ \\
\hline Initial IHO-S & $1.4 \pm 0.6$ & 0.0001 \\
Final IHO-S & $0.9 \pm 0.3$ & \\
Initial GBI & $46.3 \pm 19.9$ & 0.0005 \\
Final GBI & $21.5 \pm 7.5$ & \\
Initial DMFT & $12.2 \pm 9.4$ & 0.2500 \\
Final DMFT & $12.7 \pm 9.6$ & \\
\hline
\end{tabular}

Wilcoxon test (5\% significance level), SD: Standard deviation

The means, standard deviations and statistical differences obtained for indexes IHO-S, GBI and DMFT, measured at the initial and final phases of the CDCP, are shown in Table 2. A statistically significant difference was observed between the initial and final IHO-S and GBI indicators ( $p<0.01-W i l c o x o n$ test). No statistically significant differences were observed between initial and final DMFT ( $p>0.05$-Wilcoxon test). Therefore, CDCP implementation favored a reduction in biofilm and gingival inflammation levels (qualification of IHO-S and GBI indexes), avoiding a progression in cavity disease order (DMFT stabilization).

A significant improvement was observed in the oral hygiene of study participants after the study, as seen in the significant reduction in IHO-S, with a transition from regular to satisfactory condition (Table 2). Likewise, a significant reduction was seen in gingival bleeding for study participants (Table 2). On average, users evolved from severe gingivitis (46.3\% bleeding) to moderate gingivitis (21.5\% bleeding). The absence of a statistically significant difference between the means for initial and
Table 3: Means, standard deviations and statistical differences obtained for decayed (D), missing (M) and Filled (F) components of the DMFT index, measured at the initial and final stages of the comprehensive dental care protocol (Joao Pessoa, 2010)

\begin{tabular}{lll}
\hline Indexes & Means and SD & $\begin{array}{l}\text { Statistical differences } \\
\text { between the means }(p)\end{array}$ \\
\hline Initial D & $2.5 \pm 2.4$ & 0.0016 \\
Final D & $0.0 \pm 0.0$ & \\
Initial M & $5.0 \pm 6.8$ & 0.0975 \\
Final M & $5.6 \pm 7.8$ & \\
Initial F & $4.9 \pm 4.9$ & 0.0020 \\
Final F & $7.3 \pm 5.7$ & \\
\hline
\end{tabular}

Wilcoxon test ( $5 \%$ significance level), SD: Standard deviation

final DMFT suggests that implementation of the CDCP did not lead to a significant change in DMFT, which represents the stabilization of cavity disease (Table 2).

The means, standard deviations and statistical differences obtained for the decayed, missing and filled components of DMFT, measured at the initial and final stages of the CDCP, are shown in Table 3. A statistically significant difference was observed for components decayed and filled $(p<0.01$-Wilcoxon test). No statistical difference was observed between the initial and final Missing component ( $\mathrm{p}>0.05$-Wilcoxon test). Therefore, it is observed that the total number of missing teeth did not change significantly, whereas decayed components were turned into filled components, representing a statistically significant difference (Table 3).

\section{DISCUSSION}

During the first semester of CDCP implementation, a significant impact was observed on the oral health of the population and on the productivity of the dental service. Under the conditions set for basic care oral 
health assistance in SUS, dental surgeons must complete 40-hour work weeks, or 160 hours monthly-a longer workload than that observed in the CDCP during the period of this investigation. Therefore, it was evidenced that implementation of the CDCP in primary healthcare is a plausible proposal that can contribute to promote health in the population.

It should be emphasized that the CDCP was implemented based on weekly interventions in the routine of the basic care dental service, leading to significant changes in the health condition of users, in the access and resolution rates of the service. Thus, the consolidation and expansion of CDCP activities within the routine of oral health services in basic care could improve the dental assistance offered to users. It would be necessary to incorporate the activities set by the CDCP on a daily basis, but the increased number of work shifts would favor the expansion of access and increase resolution rates.

Conclusion of dental treatment and basic discharge were achieved by $62.5 \%$ of users, which represents a high rate of resolution for the service. Although the city administration and the profile of the assisted population can interfere in the organization of services and in defining goals to be met by SUS, ${ }^{11}$ it was observed that the indicators obtained starting with the implementation of the CDCP were better than those reported by other studies, during an evaluation of the productive aspect of SUS. ${ }^{2,11,12}$

The guidelines of the National Oral Health Policy mandate the expansion of access to dental services, so that $100 \%$ of basic care users have at least one initial dental appointment annually to define a diagnosis and treatment plan. ${ }^{1}$ However, the prevalence of first dental appointments has not surpassed the $20 \%$ mark. ${ }^{11}$ This evidences that the CDCP has the potential to contribute to the expansion of coverage and resolution of services offered by SUS.

Higher rates of permanent tooth extractions have been linked to poor access to health services and inferior oral health conditions among the population. ${ }^{11,12}$ Thus, by expanding access and resolution, as well as improving dental indicators, the CDCP contributed to reduce the rate of extractions and change the care profile, as seen in the indicators.

The results of the present study indicate 518 basic-level procedures. Of those, $51.93 \%$ consisted of preventive and promotional activities, while $48.07 \%$ involved curativerestorative treatment. This evidenced that the dental assistance offered to users to promote health was balanced between preventive and curative actions (Table 1). Moreover, the balance between promotional and restorative interventions can contribute to reduce costs and guide clinical interventions toward the real health needs of patients. ${ }^{5}$

As also observed in the literature, ${ }^{13,14}$ the DMFT value identified in this study show a high prevalence of tooth decay. However, following CDCP implementation, no statistically significant differences $(p>0.05)$ were observed between the initial and final DMFT of sample components. It was also detected that the missing component did not vary significantly ( $p>0.05)$, unlike the decayed and filled components. This demonstrates that the actions performed by the CDCP concentrated on promoting health and performing restorative procedures, aiming for recovery and preservation of dental elements. ${ }^{13,14}$

The destructive and cumulative pattern of cavity disease is the condition expected for patients that did not receive satisfactory dental assistance. ${ }^{15}$ Thus, the CDCP makes it possible to inhibit cavity disease and stabilize the number of healthy and missing elements, evidencing the impact of the actions on decayed and filled elements. Therefore, the maintenance of healthy components and the increase in filled teeth without cavities represents an indicator of the effectiveness of the service provided. ${ }^{15}$

Unlike cavity disease, gingival bleeding and biofilm accumulation on teeth are reversible conditions, which depend on patient motivation, incorporation of oral hygiene practices and elimination of bacterial niches. ${ }^{15-17}$ The literature has shown that an adequate mouth environment, health education and periodical controls at the dentist's office afforded improvements in the oral hygiene index of children assisted in health promotion programs. ${ }^{16,17}$ The CDCP also sought to reduce oral hygiene and gingival bleeding indexes to satisfactory levels, with a statistically significant difference $(p<0.05)$ between the OHI-S and GBI indexes at the initial and final stages of the CDCP. Thus, it was detected that the CDCP played a significant role in the qualification of oral indexes and in the improvement of the oral health condition of assisted users.

With regard to the presence of oral biofilm, as identified by IHO-S, a significant reduction was observed after implementation of the CDCP, reaching a mean value of 0.8 , which is considered satisfactory. ${ }^{8}$ These results indicate that the activities undertaken in the CDCP contributed to the incorporation of preventive and oral hygiene practices, as observed in the literature. ${ }^{15-17}$ As in several other studies, the present work showed that promoting oral health in a dental clinic setting, translated into the incorporation of hygiene practices and restorative treatments, contributed to improve the oral health condition of study participants. ${ }^{16-18}$ 
Given the significant reduction in GBI (from 46.3\% to $21.5 \%$ ), it was observed that gingival bleeding among users improved from severe to moderate, ${ }^{9}$ which represents an evolution of the periodontal condition. Under those conditions, basic discharge was offered considering significant improvement in the index, enabling patient oversight and motivation. The endurance of CDCP activities will strengthen the attention directed toward the periodontal condition.

It is expected that the projected periodic controls will meet the eventual needs of users and maintain the basic discharge status. Thus, future studies must consider the continuity of CDCP actions and their impact on the health of patients. The literature does not determine the actual follow-up interval necessary to maintain health levels, showing that the risk of illness changes not only with the passage of time, but rather is influenced by the care offered by the professional and by changes in habits of the patient. ${ }^{18,19}$

Improvements in the dental field within SUS must be achieved starting with programs that consider the integrality, resolution, health promotion and changes in care practices. Incorporating integral care guided by user needs made it possible for the $\mathrm{CDCP}$ to illustrate the significant change in the oral health condition and care practices of users.

\section{CONCLUSION}

It was concluded that CDCP implementation had a beneficial effect on the oral health of the population assisted by the dental services offered in basic care. The $\mathrm{CDCP}$ contributed to the improvement of oral health service care provision, besides the enhancement of volunteers' oral health and expansion of dental service discharge.

The CDCP had accomplished the public dental service demands and should be employed in primary care oral health services in order to upgrade this sector, achieve access enlargement and promote public oral health.

\section{REFERENCES}

1. Brasil, Ministério da Saúde. Coordenação Nacional de Saúde Bucal. Política Nacional de Saúde Bucal. Brasília; 2004.

2. Baldani MH, Fadel CB, Possamai T, Queiroz MGS. A inclusão da odontologia no Programa Saúde da Família no Estado do Paraná, Brasil. Cad Saúde Pública 2005;21(4):1026-1035.

3. Brasil. Ministério da Saúde. Projeto SB Brasil 2010. Pesquisa Nacional de Saúde Bucal 2010: Resultados Principais. Brasília; 2011.
4. Narvai PC, Frazão P, Roncalli AG, Antunes JLF. Cárie dentária no Brasil: declínio, polarização, iniqüidade e exclusão social. Rev Panam Salud Pública 2006;19(6):385-393.

5. da Silva RP, Meneghim MC, Correr AB, Pereira AC, Ambrosano GM, Mialhe EL. Variations in caries diagnoses and treatment recommendations and their impacts on the costs of oral health care. Community Dent Health 2012;29(1): 25-28.

6. Preventivos BNP. In: Bordoni N. Programa de Educacíon Continuada Odontológica No Convencional: Odontologia Preventiva-Medidas y Programas Preventivos. 2nd ed. Washington DC: Organización Panamericana de la Salud, 1993. p. 140.

7. Padilha WWN. Manual da Clínica Integrada da Universidade Federal da Paraíba. João Pessoa: UFPB, 2003. p. 12.

8. Greene JC, Vermillion JR. The simplified oral hygiene index. J Am Dent Assoc 1964;68(1):7-13.

9. Ainamo J, Bay I. Problems and proposals for recording gingivitis and plaque. Int Dent J 1975;25(4):229-235.

10. Brasil. Ministério da Saúde. Coordenação Nacional de Saúde Bucal. Pesquisa Nacional de Saúde Bucal 2010: Projeto Técnico. Brasília, 2009.

11. Fisher TK, Peres KG, Kupek E, Peres MA. Indicadores de atenção básica em saúde bucal: associação com as condições socioeconômicas, provisão de serviços, fluoretação de águas e a estratégia de saúde da família no Sul do Brasil. Rev Bras Epidemiol 2010;13(1):126-138.

12. Fernandes LS, Peres MA. Associação entre atenção básica em saúde bucal e indicadores socioeconômicos municipais. Rev Saúde Pública 2005;39(6):930-936.

13. De Marchi RJ, Hugo FN, Hilgert JB, Padilha DM. Number of teeth and its association with central obesity in older Southern Brazilians. Community Dent Health 2012;29(1): 85-89.

14. Oulis CJ, Tsinidou K, Vadiakas G, Mamai-Homata E, Polychronopoulou A, Athanasouli T. Caries prevalence of 5, 12 and 15-year-old Greek children: a national pathfinder survey. Community Dent Health 2012;29(1):29-32.

15. Gaião L, Wanderley JNB, Almeida RVD, Padilha WWN. Promovendo Saúde Bucal em uma Clínica de Ensino Odontológico. JBC J Bras Clin Odontol Integr 2005;9(49): 105-112.

16. Silveira JL, Oliveira V, Padilha WW. Evaluation of the reduction of the visible plaque index and of the gum bleeding index in a program of oral health promotion for children. Braz Oral Res 2002;16(2):169-174.

17. Cardoso AMR, Cavalcanti YW, Padilha WWN. Impact of oral health promotion program for caregivers and children with cerebral palsy. Pesq Bras Odontoped Clin Integr 2011;11(2): 223-229.

18. Norremose R, Teixeira KIR, Munari LS, Moreira NA, Magalhães CS. Manutenção Preventiva na Clínica Integrada: Necessidades de Tratamento e Intervalo do Retorno. Pesq Bras Odontoped Clin Integr 2010;10(2):279-283.

19. Beirne PV, Clarkson JE, Worthington HV. Recall intervals for oral health in primary care patients. Cochrane Database Syst Rev 2007;4:1-39. 